INCERTA SEDIS.

11. Aleurodiscus sendaiensis YASUDA (nomen) apud LLOYD, Myc. Writ. VII. Myc. Not. No. 67. p. 1162 (1922).

Nomen JAP.: Sendai Koyaku- take (mihi).

HAB. Honshū : in Gongen-no-mori prope urbem Sendai, prov. Rikuzen (leg. A. Yasuda, 24. Oct. 1900). Herb. YasudA.

DISTRIB. Endemicus.

\title{
Chrysomyxa of Japan
}

(Notes on the Melampsoraceae of Japan II*)

$\mathrm{By}$

\section{Naohide Hiratsuka}

The genus Chrysomyxa was established by F. UNGER in 1840 taking as its type species, Chrysomyxa Abietis (WALLR.) UNGER, an autoecious lepto-form having only teleutostage in its life-cycle. In 1906, J. C. ARTHUR restricted the generic character and separated those species having all spore stages as a new genus, Nelampsoropsis (SCHRÖT.) ARTH. In the present paper, the writer has treated the genus Chrysomyxa in a broad sense.

There are about twenty species of this genus have been described up to the present time, among which the following ten species are found in our country. They are: Chrysomyxa Ledi (Alb. et Schw.) DF BARy, Ch. ledicola (PeCk) LAGERH., Ch. Rhododendri (DC.) DE BARY, Ch. expansa DIET., Ch. Cassandrae (PECK et Crint.) Tranzsch., Ch. Menziesiae DietT., Ch. Pirolae (DC.) Rostr., Ch. Ramischiae Lagerh., Ch. Empetri (Perrs.) Schröt. and Ch. Abietis (Wali.r.) UNGER. Besidesthem, a new species, Chrysomyxa alpina Hirats. $\mathrm{f}$. is now added by the writer to the mycological flora of Japan. Therefore, the total number of species in our country is eleven.

In this short paper, the writer intends to enumerate these eleven species of this genus.

The writer wishes to express here his sincere thanks to Prof. Dr. S. ITô for his valuable suggestions and constant guidance.

Chrysomyxa Unger in Beitr. zur vergl. Path. p. 24, 1840; ARTHUR in Résult. Sci. Congr. Internat. Bot. Vienne (1905), p. 338,

* Notes on the Melampsoraceae of Japan I-Thekopsora of Japan (Bot. Mag. Tokyo, XIIII, p. 12-22, 1929). 
1906; N. Amer. Fl. VII, p. 690, 1925; BUBÁK, Rostpilze Böhmens, p. 171, 1908; Dietel in Engler-Prantr, Natürl. Pflanzenfam. I, 1. Abt.**, p. 39, 1897; Fischer, Ured. Schw. p. 426, 1904; Fragoso, Fl. Ibér. Ured. II, p. 289, 1925; Grove, Brit. Rust Fungi, p. 310, 1913; HARIOT, Uréd. p. 282, 1908; KLEBAHN in Kryptogamenfl. Mark Brandenbr. Va, p. 707, 1913; Migula, Krypt.-Fl. III, 1, p. 456, 1910; Oddemans, Rév. Champ. I, p. 575, 1892; Plowrigh't, Monogr. Ured. \& Ustil. p. 252, 1889; SACCARdo, Syll. Fung. VII, p. 579, 1888; Schröter, Pilze Schles. I, p. 371, 1887 ; Sydow, Monogr. Ured. IIÍ, p. 502, 1915; TrOTTTER, Fl. Ital. Crypt. Ured. p. 358, 1914; WeIR in Mycologia XV, p. 183, 1923; WINTER in Pilze Deutsch1. I, p. 249, 1881.

Syn. Coleosporium $§$ Melampsoropsis SchröT. in Beitr. Biol. Pfl. III, p. 57, 1879.

Melampsoropsis ARTH. in Résult. Sci. Congr. Internat. Bot. Vienne (1905), p. 338, 1906; N. Amer. Fl. VII, p. 117, 1907; BI.ASDALE in Univ. Calif. Pub1. Bot. VII, p. 114, 1919.

\section{Key to the Japanese species of Chrysomyxa}

Uredo-and teleutospores on Ericaceae.

Uredo-and teleutospores on Ledum.

Uredo-and teleutosori hypophyllous.

1. Chrysomyxa Ledi (Alb. et Schw.) De BARy

Uredo-and teleutosori epiphyllous.

2. Chrysomyxa ledicola (PECK.) LAGERH.

Teleutospores on Rhododendron.

Uredospores present.

Teleutosori hemispherical or conical without a narrwed short stalk. On Sect. Rhodrastrum.

3. Chrysomyxa Rhododendri (DC.) DE BARY Teleutosori expands into a subglobose or ellipsoidal head with a narrowed short stalk. On Sect. Eurhododendron.

4. Chry'somyxa alpina Hirat's. f.

Uredospores lacking, on Sect. Eurhododendron.

5. Chrysomyxa expansa DIE'T. Uredo-and teleutospores on Chamaedaphne.

6. Chrysomyxa Cassandrae (PECk et Clintr.) Tranzsch. Uredospores lacking, teleutospores on Menziesia.

7. Chrysomyxa Menziesiae DIET.

Uredo-and teleutospores on Pirolaceae.

Primary uredospores absent in its life-cycle. 
8. Chrysomyxa Pirolae (DC.) RosTr. Primary uredospores present in its life cycle.

9. Chrysomyxa Ramischiae LAGERH. Uredo-and teleutospores on Empetraceae.

10. Chrysomyxa Empetri (PERS.) SCHRÖT. Teleutospores on Pinaceae.

11. Chrysomyxa Abietis (WALLR.) UNGER

\section{On Ericaceae}

1. Chrysomyza Ledi (Al.b. et Schw.) De Bary in Bot. Zeitg. XXXVII, p. 809, pl. X, fig. 7, 8, 1879; BUBÁk, Rostpilze Böhmens, p. 172; DieTEl in Eingler-Prantr, Natürl. Pflanzenfam. I, 1. Abt.**, p. 40, 1897; Hiratsuka in Jour. Facul. Agric. Hokkaidô Imp. Univ. XXI, p. 34, 1927 ; KLEBAHN in Kryptogamenf. Mark Brandenbr. Va, p. 710; p. 692, fig. K, 2 (I-III); Liro, Ured. Fenn. p. 459; Migula, Krypt.-Fl. III, 1, p. 457; SACCARdo, Syll. Fung. VII, p. 760; Schröter, Pilze Schles. I, p. 371; Svdow, Monogr. Ured. III, p. 504; WinteE in Pilze Deutschl. I, p. 251. (Hiratsuka in Jap. Jour. Bot. III, p. 315, 1927; Bot. Mag. Tokyo, XLII, p. 32, 1928; Jour. Soc. Agric. \& Forestr. Sapporo, XIX, no. 88, p. 121, 1928 ; Transact. Sapporo Nat. Hist. Soc. X, p. 121, 1929; Hiratsuka \& Homma in Jour. Soc. Agric. \& Forestr. Sapporo, XIX, no. 85, p. 78, 1927).

Syn. Uredo Ledi Alb. et Schw., Consp. Fung. Nisk. p.125, 1805. U. abietina Spreng., Syst. Veg. IV, p. 572, 1827.

U. ovoideo-aurantiaca Bon., Coniom. et Cryptom. p. 32, 1860.

Caeoma Ledi ScHI.ECH'., F1. Berol. II, p. 122, 1824.

C. Ledi Link, Sp. P1. II, p. 15, 1825.

C. longiusculum Lasch in KLOTZSCH, Herb. myc. I, no. 495, 1842.

C. piceatum LINK, Sp. P1. II, p. 62, 1825, p. p.

Erysibe Ledi Wallr., Fl. Crypt. Germ. II, p. 199, 1833.

Pucciniastrum Ledi KarsT., Myc. Fenn. IV, p. 57, 1878.

Coleosporium Ledi ScHröт., Beitr. Biol. Pfl. III, p. 55, 1879.

Melampsoropsis abietina ARTH. in N. Amer. Fl. VII, p. 119, 1907.

M. Ledi ARTH. in Résult. Sci. Congr. Internat. Bot. Vienne (1905), p. 338, 1906.

Aecidium abietinum Al.8. et Schw., Consp. Fung. Nisk. p. 120, 1805, p.p.; ReEss in Abhandl. Naturf. Ges. Halle XI, p. 98, 1869.

Peridermium abietinum Тнӥм. in Mitteil. Forstl. Versuchsw. Oesterr. II, p. 320, 1881; ARTHUR \& KERN in Bull. Torr. Bot. Club, XXXIII, p. 430, 1906. 
Hab. On leaves of Picea jezoensis CARr. (Yezo-matsu).

S. Saghalien: Manui (Aug. 22, 1928, NaOHIDE Hiratsuka).

On leaves of Ledum palustre L. var. angustum BURSCH (Hosobaisotsutsuji).

S. Saghalien:-Mt. Tosso (July 20, 1927, NaOHide Hiratsuka, S. IWADARE, M. NAGAI \& S. ARAKI); Shisuka (Aug. 12, 1928, NAOHIDE HIRATSURA).

On leaves of Ledum palustre L. var dilatatum WAHL. (Karafutoisotsutsuji).

Hokkaidô:-Prov. Tokachi: Mt. Nupukaushi (June 6, 1925, NAOhIDE Hiratsuka). Prov. Kushiro: Mt. Meakan (July 19, 1921, K. TOGaShI); Mt. Oakan (July 14, 1928, NaOHIDE HiraTsuka); Kawayu (July 17, 1928, S. Shimada, M. 'Terui, K. Sasaki \& K. Fujita); Mt. Atosa-nupuri (July 17, 1928, S. Shimada, M. Terui, K. SASAKI \& K. Fujita). Prov. Nemuro: Ochiishi (July 16, 1924, NaOHide Hiratsuka \& I. Tanaka; July 21,1928, S. Shimada, M. Trirui, K. Sasaki \& K. FUjiTA).

S. Saghalien:-Aihama (July 17, 1927, NaOHIdE Hiratsuka, S. IwadAre, M. NAGAi \& S. Araki); Shisuka (Aug. 3, 1928, S. ShimaDa, M. Terui \& K. Fujita; Aug. 13, 1928, NaOhide Hira'suka); Manui (Aug. 20, 1928, NaOhide Hiratsuka); Mt. Kashipo (Aug. 8, 1928, NaOhide Hiratsuka, S. Shimada, M. Terui \& K. Fujita).

Kuriles:-Shikotan: Shakotan (July 17, 1924, A. ABE).

On leaves of Ledum palustre L. var. nipponicum NAKAI (Isotsutsuji).

Hokkaidô:- Prov. Ishikari : Horomui (June 14, 1925, NAOHIDE Hiratsuka \& I. Tanaka; Sept. 10, 1923, NaOhide Hiratsuka).

On leaves of Ledum palustre L. var. yesoense NAKAI (Yezo-isotsutsuji).

Hokkaidô:-Prov. Iburi: Noboribetsu (Sept. 3, 1910, K. Mryabe). Prov. Ishikari : Mt. Kuro-dake (Aug. 5, 1925, NAOHIDE Hiratsuka); Hakuun-sawa (Daisetsu-zan) (Aug. 14, 1927, S. I'rô, NAOHIDE HIRATSUKA \& S. IWADARE).

Distrib. Europe, United States of America, Siberia and Japan (Hokkaidô, Saghalien and the Kuriles).

2. Chrysomyza ledicola (PECK) LAGERH. in Tromsö Mus. Aarsh. XVI, p. 119, 1893; HiRA'TsUkA in Jour. Facul. Agric. Hokkaidô Imp. Univ. XXI, p. 35, 1927; Sydow, Monogr. Ured. III, p. 507. (HIRATSUKA in Jap. Jour. Bot. III, p. 315, 1927).

Syn. Uredo ledicola PECK in Ann. Rept. N.Y. State Mus. XXV, 
p. $90,1873$.

Puccinia Ledi Berk. et CURT. in Grev. III, p. 54, 1874; SACCARDo, Syll. Fung. VII, p. 713.

Dicaeoma Ledi Kun'rze, Rev. Gen. III, p. 469, 1898.

Melampsoropsis ledicola ARTH. in Résult. Sci. Congr. Internat. Bot. Vienne (1905), p. 338, 1906; N. Amer. Fl. VII, p. 119; FrasER in Mycologia III, p. 70, 1911; IV, p. 177, 1912.

Hab. On leaves of ledum palustre L. var. procumbens AIT. (Hime-isotsutsuji).

Hokkaidô:-Prov. Ishikari : Mt. Hokkai-dake (Aug. 4, 1925, NaOhide Hiratsuka ; Aug. 19, 1925, K. Miyabe \& NaOHide HiraTSUKA); Mt. Hakuun-dake (Aug. 5, 1925, NaOHIde HiraTsuka); Mt. Kuro-dake (July, 1928, H. KATAOKA; July 26, 1926, S. ITÔ, NAOHIDE: Hiratsuka, etc.; Aug. 4, 1925, NaOHide Hiratsuka ; Aug. 18, 1925, K. MiYabF, \& NaOHIdE Hiratsuka); Kumonotaira (Daisetsu-zan) (July 28, 1926, S. Itô, Naohide Hiratsuka, etc.; Aug. 19, 1925, K. Miyabe \& NaOHide Hiratsuka).

Distrib. United States of America, Canada, Alaska, Kamchatka, Greenland and Japan (Hokkaidố).

3. Chrysomyxa Rhododendri (DC.) DE BARY in Bot. Zeitg. XXXVII, p. 809, pl. X, fig. 1-6, 1879; BüÁk, Rostpilze Böhmens, p. 171, fig. 39 ; Dietei, in ENGler-Prantr, Natürl. Pflanzenfam. I, 1. Abt. ${ }^{* *}$, p. 39, fig. 23, 1897 ; Fischer, Ured. Schw. p. 426; Fragoso, F1. Ibér. Ured. II, p. 291, fig. 142, 143; GrovE, Brit. Rust Fungi, p. 384; Hakio'r, Uréd. p. 283, fig. 45 ; KLEBAHN in Kryptogamenfl. Mark Brandenbr. Va, p. 708; p. 692, K, 1 (I-III); Migula, Krypt.-F1. III, 1, p. 456, pl. IX, J, fig. 2; SACCARdo, Syll. Fung. VII, p. 760 ; Sydow, Monogr. Ured. III, p. 508, pl. XXIII, fig. 168; 'TroTtER, Fl. Ital. Crypt. Ured. p. 359, fig. 25, 26, 87 ; WinTer in Pilze Deutschl. I, p. 250, fig. 1, 2. (DieTEL in Engl. bot. Jahrb. XXXViI, p. 107, 1905 ; HenNINGS in Engl. bot. Jahrb. XXVIII, p. 263, 1900; XXXII, p. 36, 1902 ; Hiratsuka in Jap. Jour. Bot. III, p. 316, 1927 ; YoshinaGA in Bot. Mag. Tokyo, XV, p. (95), 1901).

Syn. Uredo Rhododendri DC. in Fl. frans. VI, p. 86, 1815.

Caeoma Rhododendri Link, Sp. P1. II, p. 16, 1825.

C. piceatum LINk, Sp. P1. II, p. 62, 1825, p.p.

Erysibe Rhododendri WallR., F1. Crypt. Germ. II, p. 199, 1833.

Coleosporium Rhododendri ScHRÖT. in Beitr. Biol. Pfl. III, p. 56, 1879.

Melampsora Rhododendr Tнӥм. in Bull. Soc. Imp. Nat. Moscou, 
LV, p. 87, 1880.

Melampsoropsis Rhododendri ARTH. in Résult. Sci. Congr. Internat. Bot. Vienne (1905), p. 338, 1906.

Aecidium abietinum ArB. et ScHw., Consp. Fung. Nisk. p. 120, 1805, p. p.

Hab. On leaves of Rhododendron indicum (L.) Sw. var. macranthum MAXIm. (Satsuki-tsutsuji).

Shikoku :-Prov. Tosa : Sakawa-machi (1906, T. YoshinaGa).

On leaves of Rhododendron dauricum L. (Yezo-murasaki-tsutsuji).

Hokkaidô:-Prov. Iburi : Mukawa (Aug. 27, 1895, S. Endô). Prov. Hidaka : Mt. Apoi (Aug. 17, 1912, K. Kondô). Prov. Nemuro : Ochiishi (July 17, 1924, Naohide Hiratsuka). Prov. Tokachi: Lake side of Shikaribetsu-numa (July 7, 1925, NAOHIDE Hira'TsukA).

Distrib. Europe, Siberia, Manchuria and Japan (Honshî, Shikoku and Hokkaidô).

4. Chrysomyxa alpina Hiratsuka, f. sp. nov.

Uredosori hypophyllous, occasionally on petioles, scattered, round, pulverulent, yellow; uredospores globose, ovate, ellipsoidal or oblong, 18-36 $\times 14.4-23.4 \mu$; epispore densely echinulate, hyaline; contents yellow.

Teleutosori hypophyllous, causing reddish yellow or brown spots on the upper surface, scattered or grouped, minute, expands into a subglobose or ellipsoidal head, $0.25-0.6 \mathrm{~mm}$. in diameter, waxy yellow; teleutospores catenulate, $0.12-0.28 \mathrm{~mm}$. long, cylindrico-prismatic, oblong to ovate, $14.4-27 \times 7.2-12.6 \mu$; epispore rather thin, smooth.

Hab. On leaves and petioles of Rhododendron chrysanthum PAI,L. (Kibana-shakunage).

Honshî:-Prov. Shinano: Mt. Yatsugatake (July 9, 1925, K. TOGASHI).

Hokkaidô:-Prov. Shiribeshi: Mt. Makkari-nupuri (July 9, 1925, K. ISE). Prov. Ishikari : Mt. Sapporo (April 20, 1924 ; June 17, 1923, NaOhide :Hiratsuka ; June 25, 1914, B. Ishida); Mt. Kuro-dake (July 26, 1926, S. ITô, NaOhide Hiratsuka, etc.; Aug. 4, 1925, NAOHIDE: Hiratsuka); Mt. Eboshi-dake (Daisetsu-zan) (Aug. 5, 1925, NAOHIDE HiraTsuKa); Kumonotaira (Daisetsu-zan) (July 5, 1928 ; Aug. 4, 1925, NaOHide Hiratsuka ; July 27, 1926, S. ITô, NAOHide Hrratsuka, etc.); Mt. Hakuun-dake (Aug. 5, 1925, NAOHIDE HirATsukA). Prov. Kitami: Mt. Rishiri (Rishiri) (Aug. 5, 1922, K. TogA$\mathrm{SHI})$.

S. Saghalien:-Mt. Tosso (July 20, 1927, Naohide Hiratsuka, 
S. Iwadare, M. Nagai \& S. Araki ; July 23, 1927, NaOhide Hira'rsuka, S. IwADARE \& M. NAGaI; July 30, 1928, NaOHIDE Hira'tsuKa, S. Shimada, M. Terui \& K. Fujita); Mt. Kashipo (Aug. 8, 1928, NaOhide Hiratsuka, S. Shimada, M. Terui \& K. Fujíta).

Kuriles:-Shikotan: Shakotan (July 16, 1924, A. ABE).

Distrib. Kamchatka and Japan (Honshî, Hokkaidô, Saghalien and the Kuriles).

Remarks:-The present species distinctly differs from the related species, Chrysomyxa expansa DirT. by the presence of the uredostage.

In 1915, K. MIYABE ${ }^{1}$ identified this fungus with the latter species. Since then, it has been also reported by K. ToGASHI ${ }^{2)}$ and the writer ${ }^{3,4,5)}$ from Hokkaidô, Saghalien and the Kuriles under the same name.

In 1914, W. TRANZSCHEL ${ }^{6)}$ recorded a Chrysomyxa on Rhododendron chrysanthum PALI. from Kamchatka as Chrysomyxa Rhododen$d r i$ (DC.) DE BARY. But, that fungus seems to be identical with this species by the presence of the uredostage and its host relation.

5. Chrysomyxa expansa DIET. in Engl. bot. Jahrb, XXVIII, p. 287, 1900; Mryabe in Bot. Mag. Tokyo, XXIX, p. 258, 1915, p.p.; Saccardo, Syll. Fung. XVI, p. 319; Sydow, Monogr. Ured. III, p. 512. (Hiratsuka in Jap. Jour. Bot. III, p. 314, 1927, p. p.; Transact. Sapporo Nat. Hist. Soc. IX, p. 234, 1927).

Syn.? Peridermium Piceae-hondoensis DIET. in Engl. bot. Jahrb. XXXIV, p. 519, 1905; SACCARDo, Syll. Fung. XXI, p. 747 ; Sydow, Monogr. Ured. IV, p. 5.

Hab. On leaves of Picea jezoensis CARr. (Yezo-matsu).

Hokkaidô:-Prov. Ishikari : Ochiai (July 28, 1912, K. MIYABE).

On leaves of Rhododendron Degronianum CARR. (Shakunage).

Honshî:-Prov. Shimotsuke: Nikkô (May 29, 1899, S. Kusano, type specimen!).

On leaves of Rhododendron Fauriae FRANCH. var. roseum NAKAI (Yezo-shakunage).

Hokkaidô:-Prov. Ishikari : Kanayama (May 10, 1917, M. TocHINAI); Ochiai (July 7, 1926, M. Okamoto; July 18, 1926, NAOHIde Hiratsuka ; July 28, 1912, K. Mryabe); Kushinai (June 20, 1907,

1) Bot. Mag. Tokyo, XXIX, p. 258, 1915.

2) Jap. Jour. Bot. II, p. 81, 1924.

3) Ibid. III, p. 314, 1927.

4) Bot. Mag. Tokyo, XLII, p. 3I, 1928.

5) Jour. Soc. Agric. \& Forestr. Sapporo, XIX, no. S8, p. 121, 1928.

6) In Eifenkin, A.A.-Plantes cryptogames de Kamtschatka, p. 553, 1914. 
NaOharu Hiratsuka ; July 18, 1926, NaOhide Hiratsuka); Mt. Kuro-dake (July 5, 1928 ; July 26 1926, NaOhide Hiratsuka). Prov. Kushiro : Mt. Meakan (July 13, 1928, NAOHIDE HiraTsukA ; July 31, 1897, T. Kawakami; July 19, 1921, K. Togashi); Mt. Oakan (July 14, 1928, Naohide Hiratsuka, S. Shimada, M. Terui, K. Sasaki $\&$ K. FujITA). Prov. Tokachi: Lake side of Shikaribetsu-numa (July 6, 1925, NaOHide Hiratsuka).

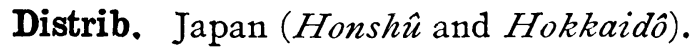

6. Chrosomyza Cassandrae (PeCk et Clint.) Tranzsch. in Trudi St. Petersb. Obshch. Est. Otd. Bot. XXIII, p. 28, 1893; HiraTSUKA in Jour. Facul. Agric. Hokkaidô Imp. Univ. XXI, p. 32, 1927; Liro, Ured.Fenn. p. 465 ; Saccardo, Syll.Fung. XVII, p. 397 ; Sydow, Monogr. Ured. III, p. 513. (HIRATSUKA in Jap. Jour. Bot. III, p. 314, 1927 ; Bot. Mag. 'Tokyo, XLII, p. 31, 1928; Hiratsuka \& Homma in Jour. Soc. Agric. \& Forestr. Sapporo, XIX, no. 85, p. 78, 1927).

Syn. Uredo Cassandrae PECK et Clint. in Ann. Rept. N. Y. State XXX, p. 54, 1878 ; SaCcardo, Syll. Fung. VII, p. 844.

Caeoma Cassandrae GoBI in Script. Bot. Hort. Univ. Petropol. I, p. 177, 1886; Gobi \& T'ranzschel in Script. Bot. Hort. Univ. Petropol. III, p. 113, 1891.

C. Cassandrae Rostr. in Medd. Bot. For. Kjöb. II, p. 90, 1888.

Melampsoropsis Cassandrae ARTH. in Résult. Sci. Congr. Internat. Bot. Vienne (1905), p. 338, 1906; N. Amer. Fl. VII, p. 119, 689; Frase in Mycologia III, p. 68, 1911; IV, p. 178, 1912.

Peridermizm consimile ARTH. et KERN in Bull. Torr. Bot. Club, XXXIII, p. 427, 1906; Saccardo, Syll. Fung. XXI, p. 746.

Hab. On leaves of Chamaedaphne calyculata (L.) MoENCH, (Horomui-tsutsuji).

Hokkaidô :-Prov. Ishikari : Horomui (June 20, 1922, NAOHIDE HIRATSUKA).

S. Saghalien :-Aihama (July 17, 1927, NAOHIDE Hiratsuka, S. IWADARE, M. NAGAI \& S. Araki); Lake side of Hakuchô-ko (July 17, 1927, NaOhide Hiratsuka, S. Iwadare, M. Nagai \& S. Araki); Makunkotan (July 29, 1928, NaOhide Hiratsuka, S. Shrmada, M. Terui \& K. Fujita); Shisuka (Aug. 2, 1927, M. NagaI; Aug. 12 \& 13, 1928, NaOHIDE Hiratsuka; Oct., 1925, S. Kagabayashi).

Distrib. Europe, Siberia, Kamchatka, United States of America, Canada and Japan (Hokkaidô and Saghalien).

7. Chrysomyxa Menziesiae DIET. in Eng1. bot. Jahrb. XXXII, p. 627, 1903; Saccardo, Syll. Fung. XVII, p. 396; Sydow, Monogr. 
Ured. III, p. 516.

Hab. On leaves of Menziesia pentandra Maxım. (Ko-yôrakutsutsuji).

Honshû:-Prov. Shimotsuke: Mt. Shirane (July 14, 1900, S. Kusano, type specimen!).

Distrib. Japan (Honshî).

\section{On Pirolaceae}

8. Chrysomyza Pirolae (DC.) Rostr. in Bot. Centralbl. V, p. 127, 1881; BƯBÁK, Rostpilze Böhmens, p. 173; DIETEL in ENGLERPranti, Natürl. Pflanzenfam. I, 1. Abt.**, p. 40, 1897 ; Fischer, Ured. Schw. p. 429, fig. 327; FraGoso, Fl. Ibér. Ured. II, p. 293, fig. 144; Grove, Brit. Rust Fungi, p. 312, fig. 236, 237 ; Hariót, Uréd. p. 282; Hiratsuka in Jour. Facul. Agric. Hokkaidô Imp. Univ. XXI, p. 36, 1927; KI,EBAHN in Kryptogamenfl. Mark Brandenbr. Va, p. 713 ; p. 722, fig. K, 4 (I-IV); Liro, Ured. Fenn. p. 456; Migula, Krypt.-Fl. III, 1, p. 457 ; Oudemans, Rév. Champ. I, p. 575; SACCARDo, Syll. Fung. VII, p. 761; Sydow, Monogr. Ured. III, p. 516; TrotTER, Fl. Ital. Crypt. Ured. p. 358. (Hiratsuka in Jap. Jour. Bot. III, p. 313, 1927 ; Transact. Sapporo Nat. Hist. Soc. IX, p. 234, 1927; X, p. 120, 1929).

Syn. Aecidium (?) Pirolae DC. in Fl. franç. VI, p. 99, 1815.

Ae. conorum-Abietis REEss in Tageb1. Vers. Deutsch. Naturf. XLII, p. 189, 1868; KarsTEN, Myc. Fenn. IV, p. 46, 1879.

Ae. conorum-Piceae REEss in Abhandl. Naturf. Ges. Halle XI, p. 102, Pl. II, fig. 1-4, 1869; FISCHER, Ured. Schw. p. 525; KLEBAHN in Kryptogamenfl. Mark Brandenbr. Va, p. 861; p. 856, fig. X, 1 (I, II) ; LIRO, Ured. Fenn. p. 564; WIN'TER in Pilze Deutschl. I, p. 260.

Ae. Engelmanni DIET. in ENGLER-Prantr, Natürl. Pflanzenfam. I, 1. Abt.**, p. 79, 1897.

Caeoma (Aecidium) Pyrolatum ScHw. in Transact. Amer. Phil. Soc. II, 4, p. 294, 1832.

Uredo pirolata KöRn. in Hedw. XVI, p. 28, 1877.

Peridermium conorum Тнӥм. in Mitteil. Forst1. Versuchsw. Österr. II, p. 313, 1881; SACCARdo, Syll. Fung. VII, p. 836.

P. Engelmanni Тнӥм. in Mitteil. Forstl. Versuchsw. Österr. II, p. $314,1881$.

P. conorum-Piceae ARTH. et $\mathrm{KERN}_{\mathrm{R}}$ in Bull. Torr. Bot. Club, XXXIII, p. 431, 1906.

Chrysomyxa pirolatum Wint. in Pilze Deutschl. I, p. 250, 1881. Ch. Pirolae Schröt., Pilze Schles. I, p. 372, 1887; Pr.OWright, 
Monogr. Ured. \& Ustil. p. 253.

Melampsoropsis Pirolae ARTH. in Résult. Sci. Congr. Internat. Bot. Vienne (1905), p. 338, 1906; N. Amer. Fl. VII, p. 118, 688.

Hab. On leaves of Pirola media Sw. (Maruba-no-ichiyakusô).

Hokkaidô:-Prov. Tokachi : Mt. Nupukaushi (June 6, 1925, NAOHIDE Hiratsuka).

On leaves of Pirola minor L. (Yezo-ichiyakusô).

S. Saghalien:-Mt. Tosso (July 30, 1928, NaOHide: Hiratsuka, S. Shimada, M. 'Terui \& K. Fujíta).

On leaves and petioles of Pirola renifolia Maxim. (Jinyô-ichiy'akusô).

Hokkaidô:-Prov. Ishikari : Sapporo (May 6, 1890, K. MiYABE); Maruyama (June 7, 1928, NaOHIDE Hiratsuka); Nopporo (May 1, 1921 ; May 8, 1920; May 18, 1922, K. Togashi ; June 16, 1923, H. Takasugi \& Y. Homma ; May 1, 1927 ; May 17, 1926; June 22, 1923; July 12, 1924, Naohide Hiratsuka). Prov. Iburi: Lake side of Shikotsu (June 5, 1927, T. Ishiyama, S. Iwadare \& M. NaGai).

Distrib. Europe, United States of America, Canada, Alaska, Greenland, Siberia, Manchuria and Japan (Hokkaidô and Saghalien).

9. Chrysomyza Ramischiae I AGERh. in Svensk Bot. Tidskr. III, p. 26, fig. 1, 3, 1909; KLEBAHN in Kryptogamenfl. Mark Brandenbr. Va, p. 715 ; Saccardo, Syll. Fung. XXI, p. 717 ; Sydow, Monogr. Ured. III, p. 518. (HIratsuka in Jap. Jour. Bot. III, p. 314, 1927).

Hab. On leaves of Pirola secunda L. (Yama-ichiyakusô).

Hokkaidô:-Prov. 'Tokachi : Lake-side of Shikaribetsu-numa (June 6, 1925, NaOhide Hiratsula).

Distrib. Europe, United States of America, Alaska, Kamchatka and Japan (Hokkaidô).

\section{On Empetraceae}

10. Chrysomyxa Empetri (Pfirs.) Scriköt., Pilze Schles. I, p. 372. 1887; Fragoso, Fl. İbér. Ured. II, p. 295; Grove, Brit. Rust Fungi, p. 311, fig. 235; Hira'suka in Jour. Facul. Agric. Hokkaidô Imp. Univ. XXI, p. 33, 1927; Liro, Ured. Fen11. p. 454; Plowright, Monogr. Ured. \& Ustil. p. 253; Migur.A, Krypt.-Fl. III, 1, p. 457; Syiow, Monogr. Ured. III, p. 515; Trot'TFR, F1. Ital. Crypt. Ured. p. 360. (Hiratsuka in Jap. Jour. Bot. III, p. 316, 1927 ; Transact. Sapporo Nat. Hist. Soc. IX, p. 234, 1927; Bot. Mag. Tokyo, XLII, p. 32,1928 ). 
Syn. Uredo Empetri PERs. in Moug. et NES'TL., Stirp. Crypt. vogeso-rhen. no. 391, 1812.

Caeoma Empetri Link, Sp. P1. II, p. 16, 1825.

C. Empetri WinT. in Pilze Deutsch1. I, p. 257, 1881.

Erysibe Empetri Wallr., Fl. Crypt. Germ. II, p. 199, 1833.

Thekopsora Empetri KarsT., Myc. Fenn. IV, p. 143, 1879.

Chrysomyxa Empetri RosTr. in Medd. Bot. For. Kjöb. III, p. 536, 1888; BubÁk, Rostpilze Böhmens, p. 73 ; Fischèr, Ured. Schw. p. 577 ; HARIOT, Uréd. p. 282; KI.EBAHN in Kryptogamenfi. Mark Brandenbr. Va, p. 716; p. 722, fig. K, 6(I, II); LAGERHEIM in Tromsö Mus. Aarsh. XVI, p. 107, 109, 1893; SaCCARDO, Syll. Fung. VII, p. 762.

Melampsoropsis Empetri ARTH. in Résult. Sci. Congr. Internat. Bot. Vienne (1905), p. 338, 1906; N. Amer. F1. VII, p. 118.

Hab. On leaves of Empetrum nigrum L. (Gankôran).

Honshû :-Prov. Rikuchû: Mt. Hayachine (Aug. 3, 1905, G. YAMADA). Prov. Shinano: Mt. Yatsugatake (Aug. 1926, G.YAMAnA).

Hokkaidô:-Prov. Ishikari: Mt. Kuro-dake (July 28, 1926, S. İTô, Naohide Hiratsuka, etc.). Prov. Tokachi : Lake side of Shikaribetsu-numa (July 7, 1925, Naohide Hiratsuka). Prov. Kushiro: Mt. Meakan (July 19, 1921, K. 'TogAshI) ; Mt. Oakan (Aug. 10, 1923, Naohide Hiratsuka). Prov. Nemuro: Nemuro (July 12, 1925, NaOhide Hiratsuka; July 19, 1924, Naohide Hiratsuka \& I. Tanaka).

S. Saghalien:-Mt. Tosso (July 23, 1927, Naohide Hiratsvka, S. Iwadare \& M. Nagar; July 30, 1928, NaOhide Hiratsuka, S. Shimada, M. Térui \& K. Fijita).

Distrib. Europe, United States of America, Greenland, Canada and Japan (Honshû, Hokkaidô and Saghalien).

\section{On Pinaceae}

11. Chrysomyxa Abietis (WALLR.) UNGER in Beitr. zur vergl. Path. p. 24, fig. 1-8, 1840; BubÁk, Rostpilze Böhmens, p. 174, fig. 40; Dietel in ENGLER-Pran'Tl, Natürl. Pflanzenfam. I, 1. Abt. ${ }^{* *}$, p. 40, 1897 ; Fischer, Ured. Schw. p. 429; Hiratsuka in Jour. Facul. Agric. Hokkaidô Imp. Univ. XXI, p. 32, 1927 ; KLEBAHN in Kryptogamenfl. Mark Brandenbr. Va, p. 712; p. 722, fig. K, 3 (I, II); Migur.A, Krypt. .F1. III, 1, p. 457 ; pl. IX, G, fig. 2-4; Saccardo, Syll. Fung. VII, p. 762 ; SchröTER, Pilze Schles. I, p. 372; Sydow, Monogr. Ured. III, p. 519; Tro'Tter, Fl. Ital. Crypt. Ured. p. 360 ; WeIr in Mycologia XV, p. 183, 1923. (Hrratsuka in Jap. Jour. Bot. III, p. 316, 1927). 
Syn. Blennoria Abietis WALI,R. in Allgem. Forst.-u. Jagdzeitg. XVII, p. 65, 1834.

Uredo epidermoidalis HARTIG in Verhandl. d. Harzer Forstverei11s,p. 61, 1864.

Sphaeria navicularis WALLR. in Tharandter Jahrb. p. 111, 1853.

Hab. On leaves of Picea Glehni MAST. (Aka-ezomatsu).

Hokkaidô:-Prov. Ishikari: Nopporo (March 5, 1918, NISHImura). Prov. Tokachi: Mt. Nupukaushi (June 6, 1925, NaOHIDE Hiratsuka).

Distrib. Europe and Japan (Hokkaidô).

Table I. Showing the distribution of the species of Chrysomyxa in Japan

\begin{tabular}{|c|c|c|c|c|c|}
\hline Species & Kurile.s & Saghalien & Hokkaidô & Honshû & $\begin{array}{l}\text { Shikoku \& } \\
\text { Kiushûu }\end{array}$ \\
\hline Chrysomyxa Ledi & + & + & + & & ! \\
\hline $\mathrm{Ch}$. ledicola & & & + & & \\
\hline Ch. Rhododendri & & & + & + & + \\
\hline $\mathrm{Ch}$. alpina & + & + & + & + & i \\
\hline Ch. expansa & & & + & + & \\
\hline Ch. Cassandrae & & + & + & & \\
\hline Ch. Menziesiae & & & & + & \\
\hline Ch. Pirolae & & + & + & & \\
\hline Ch. Ramischiae & & & $\div$ & & \\
\hline Ch. Empetri & & + & + & + & \\
\hline Ch. Abietis & & & + & & \\
\hline Total & 2 & 5 & 10 & 5 & 1 \\
\hline
\end{tabular}

Table II. Showing the distribution of the Japanese species of Chrysomyxa in the world

\begin{tabular}{|c|c|c|c|c|}
\hline Species $\quad$ Region & Japan & Asia & Furope & N. America \\
\hline Chrysomyxa I edi & + & + & + & + \\
\hline Ch. ledicola & + & + & & + \\
\hline Ch. Rhododendri & + & + & + & \\
\hline Ch. alpina & + & + & & \\
\hline Ch. expansa & + & & & \\
\hline Ch. Cassandrae & + & + & + & + \\
\hline Ch. Menziesiae & + & & & \\
\hline Ch. Pirolae & + & + & + & + \\
\hline Ch. Ramischiae & + & + & + & + \\
\hline Ch. Empetri & + & & + & + \\
\hline Ch. Abietis & + & & + & \\
\hline Total & 11 & 7 & 7 & 6 \\
\hline
\end{tabular}




\section{FUNGUS INDEX}

Chry'somyxa Abietis (WALLR.) UNGFR ......... 478

Ch. alpina Hirats. f. ....... ........473

Ch. Cassandrae (PFCK et CLINT.) TranzSCH....... 475

Ch. Empetri (PERs.) SCHRÖT.............. 477

Ch. expansa Dikт. . ...............474

Ch. Ledi (AlB. et Schw.) DE BARY . ...........470

Ch. lédicola (PECK) LAGERH. .............471

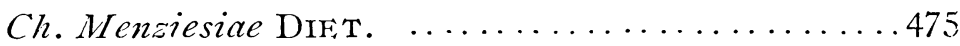

Ch. Pirolae (DC.) Rostr................ 476

Ch. Ramischiae LAGERH. ............... 477

Ch. Rhododendri (DC.) DF BARY. ..........472

\section{HOST INDEX}

Chamaedaphne calyculata (L.) MOENCH. ....

Chry'somyxa Cassandrae (PFCK et Cinte) TranzsCH. Empetrum nigrum L. ..........Ch. Empetri (PFrs.) SCHRÖT. Ledum palustre $\mathrm{I}_{1}$. var. angustum Bursch) L. palustre L. var. dilatatum WAHI. ...Ch. Ledi (ALB. et I. palustre L. var. nipponicum NAKAI SCHW.) DE BARY L. palustre L. var. procumbens AI'工. .. Ch. ledicola (PFCK) LAGFrH. 1. palustre I, var. y'esoense NAKAI ........

Ch. Leedi (Ar.B. et Schw.) DE BARY

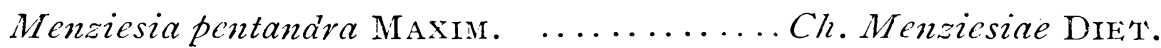
Picea Glehni MAs'T. ............Ch. Abietis (WALIR.) UNGFR P.jezoensis CARR. ......... Ch. expansa DIFT. Ch. Ledi (Aib. et Schw.) DF Bary Pirola media Sir.

P. minor $\mathrm{L}$. Ch. Pirolae (DC.) Rostr.

$P$. renifolia Maxim.

P. secunda L. ................Ch. Ramischiae LAGERH. Rhododendron chrysanthum PAI,...........Ch. alpina HIRAT'S.f. Rh. dauricum L...............Ch. Rhododendri (DC.) DF. BARY R.h. Degronianum CARR. Rh. Fauriac Franch. var. roscum NAKAI $\} \cdots .$. Ch. expansa Difi. Rh. indicum (I.) SW. var. macranthum MAxiv.

Ch. Rhododendri (DC.) DF BARY 